\title{
Risk of hyperkalemia associated with selective COX-2 inhibitors ${ }^{\dagger}$
}

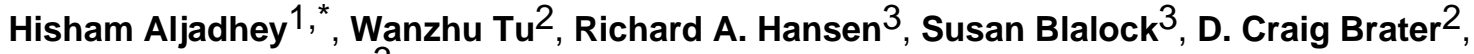 \\ and Michael D. Murray ${ }^{3}$ \\ ${ }^{1}$ Medication Safety Research Chair, College of Pharmacy, King Saud University, Riyadh, Saudi \\ Arabia \\ ${ }^{2}$ Indiana University School of Medicine, IN, USA \\ ${ }^{3}$ Division of Pharmaceutical Outcomes \& Policy, UNC Eshelman School of Pharmacy, University \\ of North Carolina at Chapel Hill, NC, USA
}

\section{SUMMARY}

\begin{abstract}
Background-Selective cyclooxygenase-2 (COX-2) inhibitors have been linked to cardiac death. The mechanism for this adverse effect appears to be by ischemic insult; however another mechanism could involve hyperkalemia. The objective of this study was to determine the effects of selective COX-2 inhibitors and non-selective nonsteroidal anti-inflammatory drugs (NSAIDs) on serum potassium concentration and the electrocardiogram.
\end{abstract}

\begin{abstract}
Methods-A retrospective cohort study was conducted using propensity score matching of patients from an inner-city academic medical center at Indianapolis, Indiana. Two hundred and two patients prescribed selective COX-2 inhibitors were matched to 202 patients prescribed nonselective NSAIDs using propensity scores methods. Outcomes included change in serum potassium concentration from baseline and the risk of an abnormal electrocardiogram.
\end{abstract}

Results-Compared to patients prescribed non-selective NSAIDs, those prescribed a selective COX-2 inhibitor had a higher risk of serum potassium increase greater than $5 \mathrm{mEq} / \mathrm{L}$ (OR, 2.56; 95\% CI, 1.03-6.36). However, patients prescribed selective COX-2 inhibitors had no greater risk of electrocardiogram abnormality (OR, 1.16; 95\% CI, 0.74-1.82).

Conclusions-Selective COX-2 inhibitors may have a greater risk of hyperkalemia than nonselective NSAIDs. This study was exploratory with small numbers of patients. Further studies are needed to confirm these results and any association with cardiovascular events.

\section{Keywords}

hyperkalemia; NSAIDs; selective COX-2 inhibitors; retrospective cohort study; propensity score

\section{INTRODUCTION}

\author{
Nonsteroidal anti-inflammatory drugs (NSAIDs) are used widely for acute and chronic \\ arthritis. Adverse gastrointestinal effects associated with NSAIDs have led to the \\ development of selective cyclooxygenase-2 (COX-2) inhibitors, which are better tolerated \\ by the gastrointestinal tract. However, selective COX-2 inhibitors, with the possible

\footnotetext{
$\dagger$ Authors declare no conflict of interest.

Copyright (C) 2010 John Wiley \& Sons, Ltd.

*Correspondence to: H. Aljadhey, Assistant Professor of Clinical Pharmacy, Director of Medication Safety Research Chair, Clinical Pharmacy Department, College of Pharmacy, King Saud University, P.O.Box 2457, Riyadh 11451, Saudi Arabia. haljadhey@ksu.edu.sa.
} 
exception of celecoxib, are associated with adverse cardiovascular events such as myocardial infarction, stroke, and sudden cardiac death.1,2 These adverse effects led to the withdrawal from the market of two selective COX-2 inhibitors, namely rofecoxib in 2004 and valdecoxib in 2005. The potential for non-selective NSAIDs to cause such cardiovascular effects continues to be debated. Although evidence suggests that selective COX-2 inhibitors increase thrombotic cardiac events, 2 other mechanisms could play a role. For example, NSAIDs are known to cause hyperkalemia, 3 which in patients with preexisting renal insufficiency or cardiovascular disease could lead to electrocardiogram abnormalities, arrhythmia, and sudden cardiac death. 4

Hyperkalemia from NSAIDs has been the subject of several case reports. $5^{-} 11$ Also, acute interventional studies have reported that NSAIDs reduce the renal excretion of potassium. $12^{-} 14$ One small uncontrolled observational study found an increase in serum potassium concentrations with indomethacin.15 However, to our knowledge, no study has examined the effects of COX-2 inhibitors compared to other NSAIDs while controlling for important confounders and determined the risk of arrhythmia. Therefore, the objective of this study was to investigate whether selective COX-2 inhibitors are associated with an increase in serum potassium concentration and abnormalities in the electrocardiogram compared to nonselective NSAIDs using propensity score methods.

\section{METHODS}

\section{Design and subjects}

This retrospective cohort study included adult patients who received their first prescription of NSAID from the general medicine practice of Wishard Health Services in Indianapolis, Indiana. Wishard is city-county health center affiliated with the Indiana University School of Medicine. We used the Regenstrief Medical Record System (RMRS) to identify eligible patients and collect data on relevant variables. The RMRS is an electronic medical record system that captures prescriptions, laboratory test results (including electrographic results), and other clinical data.16

We used a new user design that has been recently suggested to detect early-onset adverse effects. 17 The index date was defined as the date of the first NSAID prescription between 1993 and 2006. Patients did not have an active prescription for any NSAIDs during the year preceding the starting index date. The study was approved by the Institutional Review Boards at the University of North Carolina at Chapel Hill and Indiana University-Purdue University at Indianapolis.

\section{Data analysis}

Serum potassium concentrations and electrocardiogram results were collected within the years before and after the start date of the first prescription of an NSAID. Information was collected on relevant confounders at baseline. The end date of follow-up was defined as 1 year after the first prescription or 30 days after the last dispensed prescription, whichever was less.

Propensity score matching was used to minimize selection bias by balancing covariates between comparison groups of patients prescribed COX-2 or non-selective NSAIDs. Propensity scores were calculated using SAS PROC LOGISTIC (SAS Institute, Inc., Cary, North Carolina). Mahalanobis metric matching without replacement was used since it produced good balance in covariates between comparison groups.18 A caliper of onequarter of the standard deviation of the propensity score was used in the match. To assess the covariate balance, we used $\chi^{2}$ tests for categorical variables and $t$-tests for continuous variables. 


\section{Endpoint}

Outcomes included the absolute change in serum potassium concentration before and after the index date and the risk of experiencing a serum potassium concentration $>5 \mathrm{mEq} / \mathrm{L}$. The first potassium concentration after the index date was selected because the effects of NSAIDs on renal function (including their effect on potassium homeostasis) happen within days to a week.12 In addition, physicians may alter treatments based on any increase in potassium. The secondary outcome of the study was the risk of an abnormal electrocardiogram (EKG) after receipt of NSAID. An abnormal EKG was defined as any deviation from normal as interpreted by the physician.

\section{Covariates}

Based on previous literature, the models included covariates likely to affect serum potassium concentrations or the use of NSAIDs including age, race, gender, and baseline serum potassium concentration. Models controlled for the following diagnoses: rheumatoid arthritis, osteoarthritis, coronary artery disease or myocardial infarction, stroke or transient ischemic attack, arrhythmia, asthma or chronic obstructive pulmonary disease, renal insufficiency, cirrhosis with ascites, systemic lupus erythematosus, diabetes mellitus, and congestive heart failure. To minimize bias introduced by variations in the time between baseline serum potassium concentration and the index date, time was included in the model. The analysis controlled for the use of medications known to be associated with an increase in potassium concentration including angiotensin-converting enzyme inhibitors, angiotensin II antagonists, diuretics, and potassium supplements. After matching on propensity score, a variable was included in the final model to control the time from the index date to the first measurement of serum potassium in order to reduce the potential for a temporal measurement bias.

\section{RESULTS}

A total of 1985 patients were prescribed selective COX-2 inhibitors $(n=213)$ or nonselective NSAIDs $(n=1772)$ and met inclusion criteria. Baseline characteristics differed between patients in the selective COX-2 inhibitor group compared to those receiving nonselective NSAIDs (Table 1). Patients receiving selective COX-2 inhibitors were more likely to be older, a woman, and have a diagnosis of hypertension or coronary artery disease. Based on their propensity scores, 202 patients from the selective COX-2 inhibitor group were matched to 202 patients from the group receiving non-selective NSAIDs. Table 1 shows that propensity score matching resulted in similar covariate distributions between the two treatment groups. Patients had a mean age of $62 \pm 12$ years and $84 \%$ were women.

Mean potassium concentrations (SD) before and after the index date for the selective COX-2 inhibitor group were $4.15 \pm 0.48 \mathrm{mEq} / \mathrm{L}$ (range: $3.0-5.7$ ) and $4.25 \pm 0.56 \mathrm{mEq} / \mathrm{L}$ (range: 2.9-6.2) after the index date and for the non-selective NSAIDs these values were $4.07 \pm$ $0.45 \mathrm{mEq} / \mathrm{L}$ (range: $2.7-5.3$ ) and $4.05 \pm 0.49 \mathrm{mEq} / \mathrm{L}$ (range: $2.4-5.7$ ). Mean absolute prepost change in potassium concentrations $( \pm \mathrm{SD})$ in the selective COX-2 inhibitor group was $0.09 \pm 0.57 \mathrm{mEq} / \mathrm{L}$ (range: $-1.7-2.0$ ) and for the non-selective NSAIDs the mean change in serum potassium was $-0.01 \pm 0.53 \mathrm{mEq} / \mathrm{L}$ (range: $-1.5-1.7$ ).

Crude estimates, before using the propensity score methods, indicated that the risk of increased potassium concentration was not statistically different between groups (Table 2). However, after controlling for background characteristics using propensity score matching, patients who had been prescribed a selective COX-2 inhibitor had a mean estimated increase in serum potassium concentration of $0.15 \mathrm{mEq} / \mathrm{L}(p=0.002)$ compared with patients prescribed non-selective NSAIDs. Serum potassium concentrations $>5 \mathrm{mEq} / \mathrm{L}$ occurred in 
17 patients prescribed selective COX-2 inhibitors and seven patients prescribed nonselective NSAIDs (OR, 2.56; 95\%CI, 1.03-6.36). However, patients prescribed selective COX-2 inhibitors had no greater risk of abnormal EKG compared to those prescribed nonselective NSAIDs (OR, 1.16; 95\%CI, 0.74-1.82; $n=62$ in the selective COX-2 inhibitors group and $n=56$ in the non-selective NSAIDs).

\section{DISCUSSION}

This is the first observational study to explore the association between selective COX-2 inhibitors and increased serum potassium concentrations using the propensity score matching method. The results suggest that patients prescribed selective COX-2 inhibitors may have a greater risk for increased serum potassium concentrations compared with patients prescribed non-selective NSAIDs; however, there was no increased risk of arrhythmia.

The hypothesized mechanism for hyperkalemia associated with NSAIDs is related to the inhibition of prostacyclin. 4 In contrast to COX-1, COX-2 mediates prostacyclin synthesis, which increases potassium secretion at the distal tubule.4 Thus, selective inhibition of COX-2 at the distal tubule could explain the greater risk of hyperkalemia associated with selective COX-2 inhibitors compared with non-selective NSAIDs.

This study has several limitations that should be considered when interpreting the results. Patients were from a single health system that may not be generalizable to other practices. Because of small sample sizes, the study was not powered to detect between-group differences with respect to electrocardiogram abnormalities. Hence, this study should be replicated in other settings and with greater numbers of patients.

Since the database used in this study captures only prescription NSAID, bias could be introduced if patients were classified as non-users while they are using over-the-counter NSAIDs. However, because patients included in this study were provided with their medications (including those available over the counter) through a prescription assistance program, it is less likely that they would have purchased over-the-counter NSAIDs. Furthermore, a recent sensitivity analysis suggested that missing over-the-counter drug exposure is not a significant source of bias in studies such as ours.19

If confirmed by larger studies in other settings, the results of this study could have important clinical and policy implications. Before prescribing selective COX-2 inhibitors, physicians would need to balance the benefits of medication against the risk adverse effects including hyperkalemia. Patients prescribed selective COX-2 inhibitors who have a predisposing risk of hyperkalemia or cardiovascular morbidity would need to have their serum potassium concentration monitored soon after treatment was initiated.

In summary, patients prescribed selective COX-2 inhibitors are at a greater risk for clinically important increases in potassium serum concentrations compared with patients using nonselective NSAIDs. Prescribers should consider monitoring serum potassium concentrations in patients prescribed selective COX-2 inhibitors who are at risk of hyperkalemia or cardiovascular events. Studies with greater numbers of patients should be conducted in other patient populations to confirm the results of this study.

\section{KEY POINTS}

- Hyperkalemia was associated with selective COX-2 inhibitors; however, there was no increased risk of arrhythmia 
- Prescribers should consider monitoring serum potassium concentrations in patients prescribed selective COX-2 inhibitors who are at higher risk of hyperkalemia

- Future studies with larger numbers of patients should be conducted in other patient populations to confirm the results of this study

\section{REFERENCES}

1. Graham DJ, Campen D, Hui R, et al. Risk of acute myocardial infarction and sudden cardiac death in patients treated with cyclo-oxygenase 2 selective and non-selective non-steroidal antiinflammatory drugs: nested case-control study. Lancet. 2005; 365(9458):475-481. [PubMed: 15705456]

2. Bresalier RS, Sandler RS, Quan H, et al. Cardiovascular events associated with rofecoxib in a colorectal adenoma chemoprevention trial. N Engl J Med. 2005; 352(11):1092-1102. [PubMed: 15713943]

3. Brater DC. Renal effects of cyclooxygyenase-2-selective inhibitors. J Pain Symptom Manage. 2002; 23(4):S15-S20. [PubMed: 11992745]

4. Khanna A, White WB. The management of hyperkalemia in patients with cardiovascular disease. Am J Med. 2009; 122:215-221. [PubMed: 19272479]

5. Nielsen EH. Hyperkalaemic muscle paresis-side-effect of prostaglandin inhibition in a haemodialysis patient. Nephrol Dial Transplant. 1999; 14(2):480-482. [PubMed: 10069220]

6. Haragsim L, Dalal R, Bagga H, Bastani B. Ketorolac-induced acute renal failure and hyperkalemia: report of three cases. Am J Kidney Dis. 1994; 24(4):578-580. [PubMed: 7942813]

7. Patel P, Mandal B, Greenway MW. Hyperkalaemic quadriparesis secondary to chronic diclofenac treatment. Postgrad Med J. 2001; 77(903):50-51. [PubMed: 11123398]

8. Hay E, Derazon H, Bukish N, Katz L, Kruglyakov I, Armoni M. Fatal hyperkalemia related to combined therapy with a COX-2 inhibitor, ACE inhibitor and potassium rich diet. J Emerg Med. 2002; 22(4):349-352. [PubMed: 12113843]

9. Braden GL, O'Shea MH, Mulhern JG, Germain MJ. Acute renal failure and hyperkalaemia associated with cyclooxygenase-2 inhibitors. Nephrol Dial Transplant. 2004; 19(5):1149-1153. [PubMed: 14993496]

10. Lam Q, Schneider HG. Hyperkalaemia with cyclooxygenase-2 inhibition and hypoaldosteronism. Intern Med J. 2005; 35(9):572-573. [PubMed: 16105165]

11. Kelley M, Bastani B. Ketorolac-induced acute renal failure and hyperkalemia. Clin Nephrol. 1995; 44(4):276-277. [PubMed: 8575131]

12. Murray MD, Lazaridis EN, Brizendine E, Haag K, Becker P, Brater DC. The effect of nonsteroidal antiinflammatory drugs on electrolyte homeostasis and blood pressure in young and elderly persons with and without renal insufficiency. Am J Med Sci. 1997; 314(2):80-88. [PubMed: 9258209]

13. Swan SK, Rudy DW, Lasseter KC, et al. Effect of cyclooxygenase-2 inhibition on renal function in elderly persons receiving a low-salt diet. A randomized, controlled trial. Ann Intern Med. 2000; 133(1):1-9. [PubMed: 10877734]

14. Niccoli L, Bellino S, Cantini F. Renal tolerability of three commonly employed non-steroidal antiinflammatory drugs in elderly patients with osteoarthritis. Clin Exp Rheumatol. 2002; 20(2):201207. [PubMed: 12051399]

15. Zimran A, Kramer M, Plaskin M, Hershko C. Incidence of hyperkalaemia induced by indomethacin in a hospital population. Br Med J (Clin Res Ed). 1985; 291(6488):107-108.

16. Murray MD, Smith FE, Fox J, et al. Structure, functions, and activities of a research support informatics section. J Am Med Inform Assoc. 2003; 10(4):389-398. [PubMed: 12668695]

17. Ray WA. Evaluating medication effects outside of clinical trials: new-user designs. Am J Epidemiol. 2003; 158(9):915-920. [PubMed: 14585769]

18. Baser O. Too much ado about propensity score models? Comparing methods of propensity score matching. Value Health. 2006; 9(6):377-385. [PubMed: 17076868] 
19. Yood MU, Campbell UB, Rothman KJ, et al. Using prescription claims data for drugs available over-the-counter (OTC). Pharmacoepidemiol Drug Saf. 2007; 16(9):961-968. [PubMed: 17654746] 


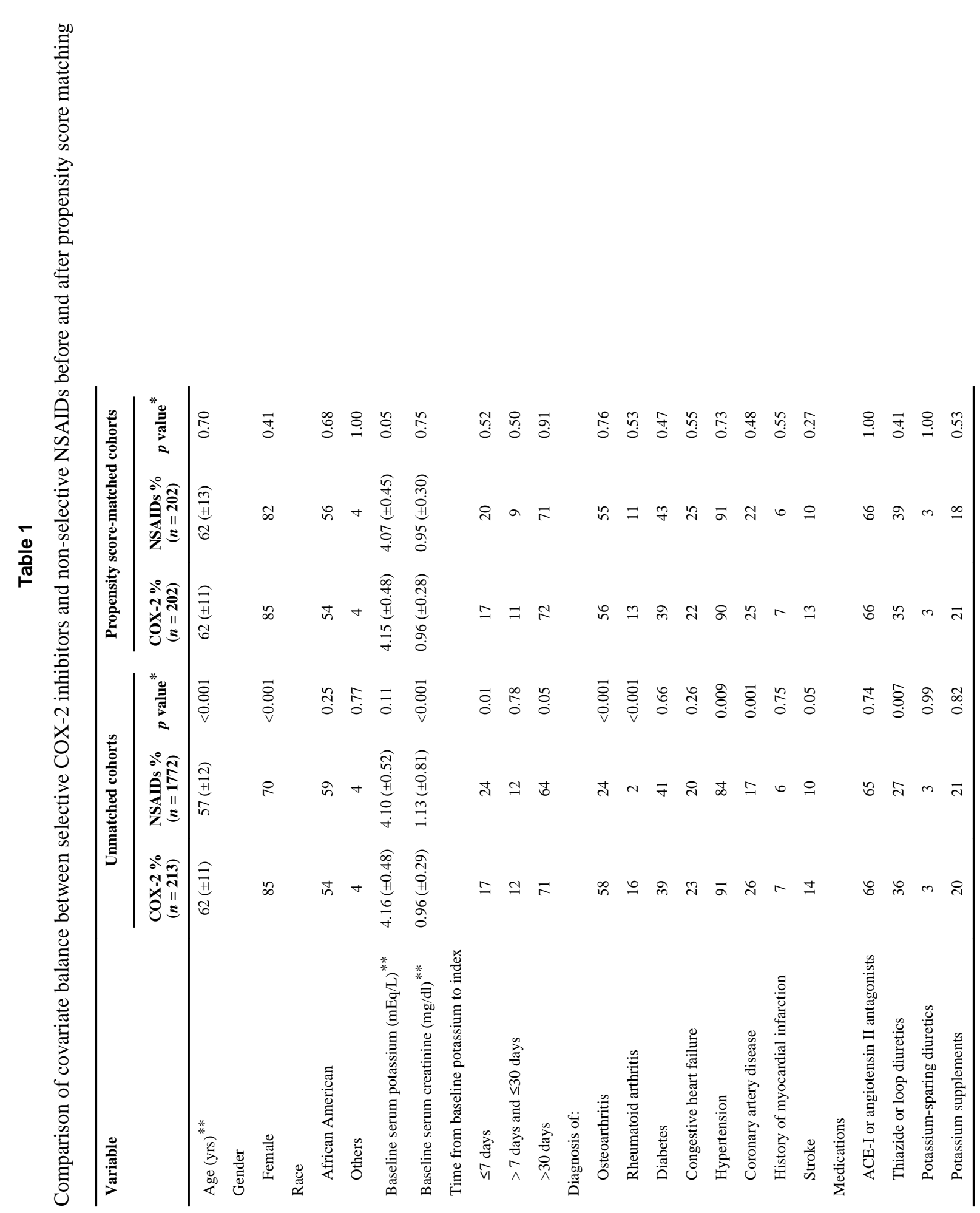


Table 2

Risk of outcomes for selective COX-2 inhibitors compared to non-selective NSAIDs

\begin{tabular}{|c|c|c|c|}
\hline Outcome & $\begin{array}{l}\text { Odds } \\
\text { ratio }\end{array}$ & $\begin{array}{c}95 \% \\
\text { confidence } \\
\text { interval }\end{array}$ & $\underset{\text { value }}{p}$ \\
\hline \multicolumn{4}{|l|}{ Unadjusted analysis $(n=1985)$} \\
\hline Incidence of $K \geq 5.0 \mathrm{mEq} / \mathrm{L}^{*}$ & 1.57 & $0.92-2.68$ & 0.10 \\
\hline Risk of abnormal EKG & 1.03 & $0.76-1.40$ & 0.86 \\
\hline \multicolumn{4}{|l|}{ Propensity score matching $(n=404)$} \\
\hline Incidence of $K \geq 5.0 \mathrm{mEq} / \mathrm{L}^{*}$ & 2.56 & $1.03-6.36$ & 0.04 \\
\hline Risk of abnormal EKG & 1.16 & $0.74-1.82$ & 0.51 \\
\hline
\end{tabular}

Incidence of potassium concentration of $\geq 5.0 \mathrm{mEq} / \mathrm{L}$ after the index date. Incident subjects were those who had baseline potassium concentration of $<5.0 \mathrm{mEq} / \mathrm{L}$ and first potassium concentration of $\geq 5.0 \mathrm{mEq} / \mathrm{L}$ after the NSAID was started. 